\title{
Performance and Results from a Space Borne, Uncooled Microbolometer Array Spectral Radiometric Imager
}

\author{
James D. Spinhirne, V. Stan Scott \\ NASA Goddard Space Flight Center/912 \\ Greenbelt, MD 20771 \\ $301-614-6274$ \\ spinhirne@gsfc.nasa.gov \\ Redgie S. Lancaster , Kathrine Manizade and Steven P. Palm \\ Science Systems and Applications Inc \\ 5900 Princess Garden Parkway \\ Lanham, MD MD 20706
}

\begin{abstract}
The Infrared Spectral Imaging Radiometer experiment was flown on a space shuttle mission as a shuttle hitchhiker experiment in August of 1997. The goals of the experiment were to test uncooled array detectors for infrared spectral imaging from space and to apply for the first time retrieval from space of brightness temperatures of cloud, land and sea along with direct laser measurements of cloud top height. The instrument operates in 3 narrow and one broad spectral band, all between 7 and 13 microns in either stare or time-delay and integration mode. The nominal spatial resolution was $1 / 4$ kilometer. Using onboard calibrations along with periodic views of deep space, radiometric calibration of imagery was carried out and performance analyzed. The noise equivalent temperature difference and absolute accuracy reported here varied with operating mode, spectral band and scene temperature but were within requirements. This paper provides a description of the instrument, its operating modes, the method of brightness temperature retrieval, the method of spectral registration and results from the flight.
\end{abstract}

\section{TABLE OF CONTENTS}

1. INTRODUCTION

2. INSTRUMENT

3. EXPERIMENTAL

4. ANALYSIS OF PERFORMANCE

5. IMAGING RESULTS

6. SUMMARY

7. REFERENCE

\section{INTRODUCTION}

We report the results for the flight of the Infrared Spectral Imaging Radiometer (ISIR) on NASA's Space
Shuttle mission STS-85 in August 1997. ISIR is the first space-flight instrument to use an uncooled microbolometer array (MBA). The instrument obtains radiometrically calibrated earth imagery in several narrow spectral bands in the thermal infrared. The purpose of the ISIR program was to demonstrate and test the applicability of uncooled MBA detectors to spaceborne radiometric sensors and to apply retrieval of thermal IR brightness temperatures of clouds, land and ocean in several selected spectral bands at high, 1/4 kilometer, spatial resolution. ISIR's scientific mission was primarily to image clouds from Shuttle orbit, giving such information as cloud top temperature and classification and discriminating cirrus cloud particle type by split window analysis. In particular during the STS-85 mission, ISIR was flown alongside the Shuttle Laser Altimeter (SLA), a Goddard laser altimeter, which provided direct vertical cloud height information. The overall goal of the ISIR program was to explore the technology of using uncooled detector arrays in a IR sensors and realize the potential for more compact and low cost systems.

There are a variety of requirements for imaging from low earth orbits. One current requirement of an operational, earth-sensing, visible and infrared imager are defined by the current Advanced Very High Resolution Radiometer (AVHRR) specifications [1,2]. AVHRR's high temporal frequency of observations, coupled with a swath of $\pm 55^{\circ}$ off-nadir, results in global imaging about once per day. There have been five bands on the original instruments, two visible/near visible channels, two splitwindow thermal infrared channels and a $3.7 \mu \mathrm{m}$ channel. The wavelength resolution is fairly broad, ranging from 100 nanometers for the visible channel and one micron for the thermal channels. The instantaneous field of view (IFOV) in the visible bands gives an approximate resolution of $1 \mathrm{~km}$ at nadir. The thermal channel spatial resolution is approximately $4 \mathrm{~km}$. In another requirement, the Moderate Resolution Imaging Spectroradiometer (MODIS) instrument developed for NASA's Earth 


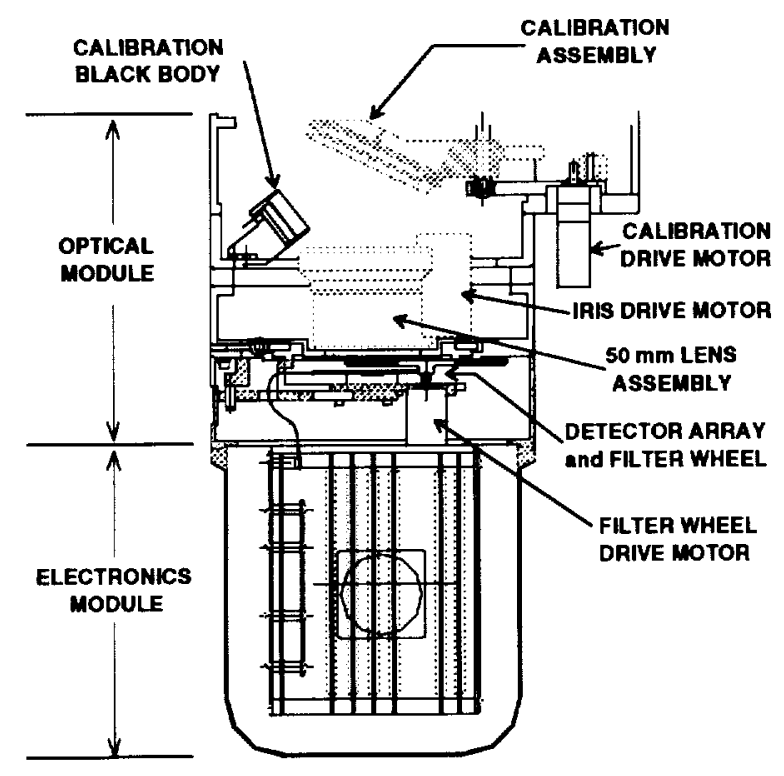

Fig. 1 Design of the imaging radiometer

Observing System (EOS) has 36 bands with $1 / 4 \mathrm{~km}$ resolution for the visible bands. MODIS, however, is a large, expensive instrument with enormous data rates [3]. We see the basic requirement for a low-earth-orbit (LEO) imager as one with possibly incremental increases in spatial resolution or number of bands over current AVHRR instruments. Higher spectral resolution of bands may improve analysis but without increasing data rates. A compact imager of such requirements would have application on any number of possible multisensor missions, and a polar-orbiting instrument similar to ISIR could be designed by a reasonably straightforward extension of the design flown on STS-85. To obtain a $3000 \mathrm{~km}$ swath-width from polar orbit with present MBA technology would require the use of several adjacent MBAs, although future array development may produce single arrays large enough to provide the larger swath width.

There are however fundamental questions on the use of an infrared array detector based instrument for LEO imaging. These include meeting sensitivity requirements, calibration and the accuracy of multi spectral pixel registration. The techniques and issues for all these are very different than for older style imaging radiometers which employ spinning scan mirrors for spatial coverage. The ISIR experiment was intended to address the accuracy, calibration and pixel registration of a uncooled focal plain array imager. Since the shuttle flight experiment, the accuracy and processing of data has been addressed. In this paper we describe the design of the instrument and report on the accuracy of the measurements and data registration.

\section{INSTRUMENT}

The MBA detector [4] is a large-format $(327 \times 240)$ silicon microbolometer array providing good sensitivity (D* 5x109 cm Hz1/2/Watt) in the thermal IR at room temperature. MBA detectors were developed in the late 1980 's and early 1990's. The Honeywell Sensor and System Development Center developed the MBA detector, using silicon micro-machining techniques to produce a monolithic array of silicon microbolometers and CMOS readout circuitry. Honeywell's MBA had 80,000 pixels (336 x 240) with average room-temperature sensitivity (NETD) of less than $0.05 \mathrm{~K}$, measured at a $30 \mathrm{~Hz}$ frame rate, with $\mathrm{f} / 1.0$ optics and a broadband ( 8 to $14 \mathrm{um}$ ) filter. In 1993 Honeywell demonstrated a nearly theoretical reduction in noise with frame averaging, suggesting MBAs would display similar noise reduction with frame integration techniques and spatial averaging. In 1994 Honeywell licensed MBA detector technology to Loral and others who are now providing low-cost commercial versions of the MBA. In 1996, Loral provided the ISIR

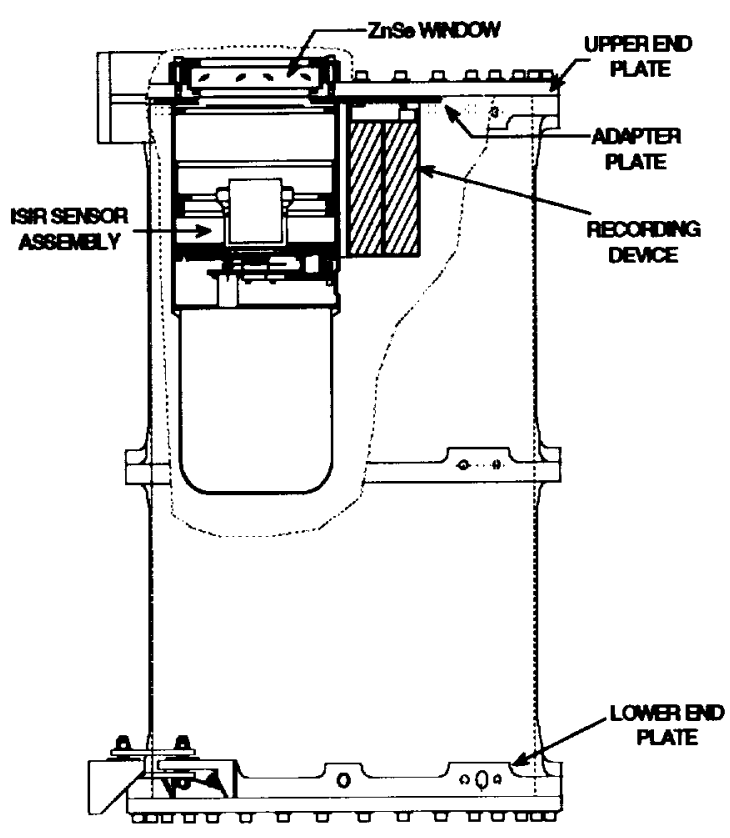

Fig. 2 Installation of imaging radiometer and data system in shuttle hitchhiker containor

project with one of its first prototype MBAs for use in the ISIR focal plane.

Prior to the ISIR program, no real assessment had been made of the spectral-radiometric performance of MBAs. All previous tests of sensitivity (using NETD and minimum resolvable temperature difference, MRTD) had 
been done broadband, because there had as yet been no applications for MBAs other than in night-vision cameras, for which narrow band sensitivity is irrelevant.

The basic optical head of the ISIR instrument was designed and built by Space Instruments, Incorporated (SII) for Goddard Space Flight Center under the Small Business Innovative Research program. ISIR was designed to fly in low earth orbit as a Shuttle Hitchhiker payload (the Hitchhiker is a small attached payload for which minimal astronaut involvement is required). The instrument is a compact spectral imager providing radiometrically calibrated infrared imagery in three one-micron wide spectral bands centered at $8.55,10.8$ and 11.8 microns and in a broad-band window from 7 to 13 microns. Test data on previously built MBA's and design calculations for ISIR indicated that NETD on the order of $0.12 \mathrm{~K}$ (the cited AVHRR performance standard) at $8.55,10.8$ and 11.8 microns was achievable. ISIR's spatial resolution requirement was $250 \mathrm{~m}$ with a total image swath-width of $90 \mathrm{~km}$ and an IFOV of 0.9 milliradians.

In order to obtain sufficient measurement accuracy, frame-averaging and time-delay-and-integration (TDI) to improve the overall signal to noise ratio (SNR) was necessary. In TDI, the signal originating from a single IFOV is integrated by pixels in successive rows of the focal

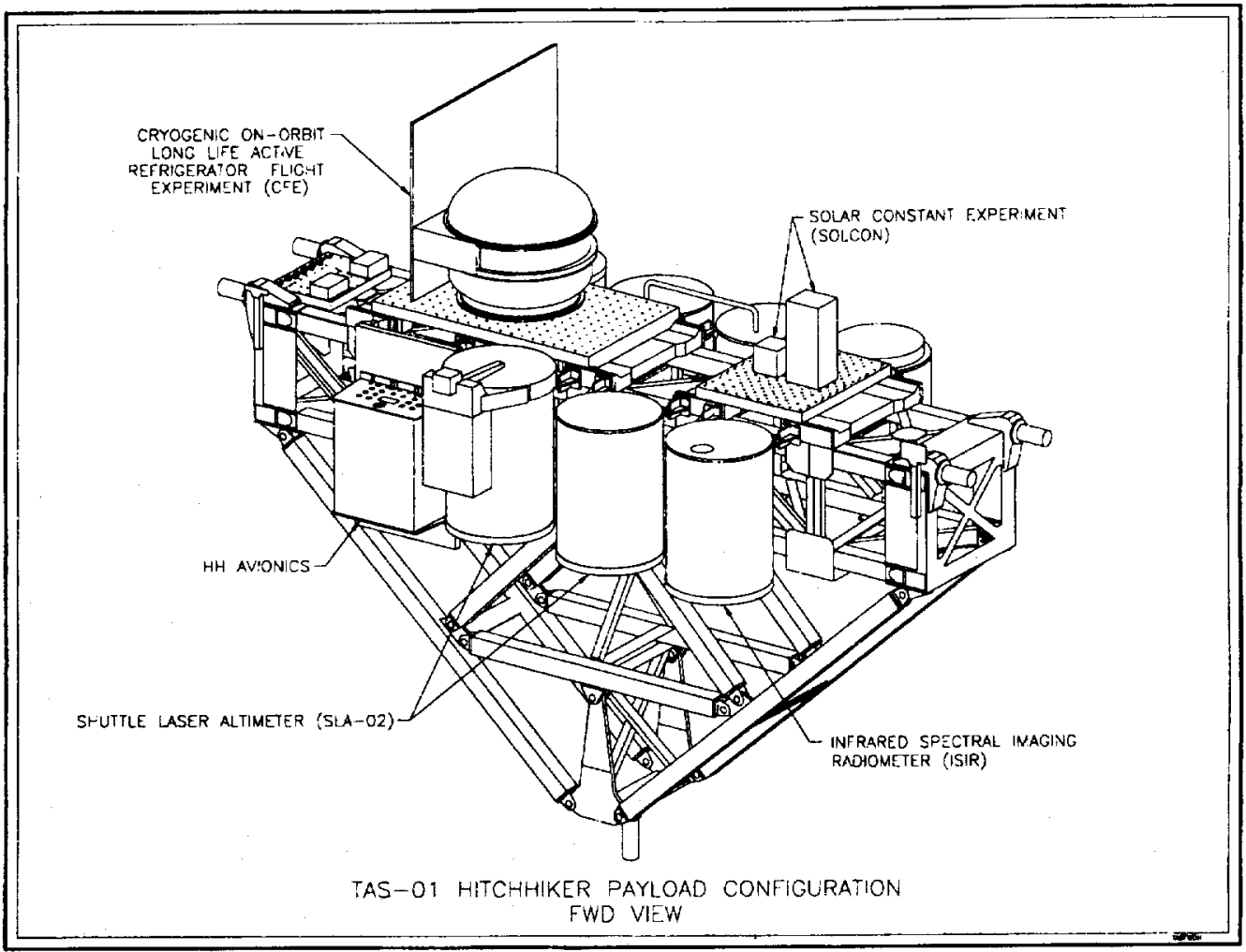

Fig. 3 The ISIR and SLA experiments as installed on a Shuttle Hitchhiker cross bay bridge. In addition the bridge carried four other hitchhikers and on STS-85 there were a total of fifteen experiments. plane as the Shuttle sweeps along its ground track. The signals from successive pixels are co-added and in the absence of correlated noise, this improves the SNR by the square root of the number of pixels in TDI. ISIR was designed to be flexible, operating either with 10 or 40 rows in TDI, or in stare-mode. In stare-mode, ISIR was capable of averaging 10 or 40 frames directly as well as acquiring only single frames.

ISIR's basic mechanical design is shown in Figure 1. Its configuration inside the Hitchhiker canister is shown in Figure 2. ISIR's IFOV is 0.9 milliradians and pixel size is $240 \times 240 \mathrm{~m}$ (at nadir) from a typical Shuttle altitude during STS-85 of 140 nautical miles. Its optical axis is defined by the center of the focal place and that of a one inch thick, 6.75-inch diameter zinc selenide ( $\mathrm{ZnSe}$ ) IR window mounted in the upper end plate of the Hitchhiker canister. The scene is viewed through the $\mathrm{ZnSe}$ window in the Hitchhiker canister (maintained with dry nitrogen at atmospheric pressure throughout the mission). A fast, f $/ 0.73,50 \mathrm{~mm}$ focal-length compound lens optimized for the 8 to 14 micron waveband images directly onto the focal plane. Spectral selection is achieved by means of a rotating filter wheel containing four band-pass filters. The filter wheel is mounted directly above the focal plane. The four spectral filters are 1.0 inch diameter germanium/ZnSe multi-layer interference filters. Because the 1.0 micron spectral bandwidths are not particularly narrow, the effects of the fast optical system on band center, shape and width of the three narrow band filters were calculated to be in acceptable limits. The MBA is covered with a thin germanium window which seals the package hermetically.

ISIR's onboard calibration system was designed to provide one- or two-point calibrations during routine operation. There is an onboard blackbody, whose temperature is controlled by a stack of thermoelectric coolers. It is mounted off-axis and during calibrations a moveable calibration mirror projects its image 
(out of focus) through the lens assembly onto the focal plane. During data-takes the calibration mirror is stowed off-axis. The overall optical transmission in any band is the product of the transmissions of all the transmitting elements in either calibration or data modes (during calibrations the $\mathrm{ZnSe}$ window is obscured from the view of the array by the calibration mirror).

The ISIR electrical system consists of the electronics assembly and drive motors along with the necessary wiring connectors and cable harnesses to connect these subassemblies to each other and to the data tape recorder, and to the Hitchhiker remote interface unit (HRIU). ISIR, as a Hitchhiker payload, was provided by the HRIU with electrical power for the sensor and the auxiliary heaters, and the signal interface with the ground station. The HRIU signal interface provided a bi-level command to power ISIR on and off, an asynchronous low-rate data stream conveying operational commands up to ISIR and housekeeping data down to the ground station, and a medium-rate data link for relaying image data to the ground station.

The readout electronic were based around a LIRIS Prototype Uncooled IR Imager detector/camera module It is housed directly above the optics module, and directly below the electronics assembly housed in a compact electronics module. The ISIR electronics assembly consists of 7 printed-circuit boards and two camera-electronics signal processing boards. The Integrator Board provides the interface with the detector module electronics and performs real-time TDI and frame averaging. The Calibration Processor Board contains a V25 microprocessor and memory to calculate and store the gain and offset coefficients for each pixel in each spectral band. The I/O Processor Board contains the I/O V25 microprocessor and the control and data interfaces. The Hardware Driver Board contains the drivers for the filter wheel stepper motor, the calibration mirror motor, the electronics-housing fan, and the blackbody. Two Power Supply Boards generate the required voltage forms from the $28 \mathrm{~V}$ input power provided by the HRIU.

Four auxiliary, 20 Watt Kapton strip heaters were mounted in the Hitchhiker canister to maintain the temperature above $0 \operatorname{degC}$ on orbit. ISIR housekeeping data was recorded by the data tape recorder, and periodically telemetered down to the ground station during the STS-85 mission. Housekeeping data included voltages, currents, and the temperatures at 21 points inside the sensor that were monitored by thermistors located in instrument subassemblies. The ISIR housekeeping temperatures are used to calculate the instrument radiation background.

The ISIR data system consists of two large-capacity $8 \mathrm{~mm}$ tape recorders (Exabyte-8505C) capable of storing 14GBytes of data when compressed at an average $2: 1$ compression ratio. This tape recorder was space-qualified by the manufacturer to withstand the stresses of launch and landing. ISIR data was also relayed to the ground station during the STS-85 mission via the medium-rate data link provided by the HRIU. ISIR data rates range from 83 to $246 \mathrm{KBytes} / \mathrm{sec}$ depending on the number of rows in TDI and the operating mode.

\section{EXPERIMENTAL}

In each mode and spectral band, laboratory and flight measurements were made of: ISIR system response (pixel gains and offsets); response linearity; SNR and NETD, signal drift over time, and spatial uniformity after gain and offset corrections. Calibration and performance data and scientific imagery were obtained in the following modes and formats:

1. Single $327 \times 240$ pixel frames in camera mode

2. Averages of 10 and $40,327 \times 240$ frames in camera mode ("Cam-10" and "Cam-40" modes respectively)

3. Push-broom images with either 10 or 40 rows in TDI ("TDI-10" and "TDI-40" respectively). The TDI-10 images were comprised of $327 \times 90$ pixels, and TDI-40 images of $327 \times 204$ pixels.

Pre and post flight laboratory tests and analysis were an important part of the mission. In the various modes and spectral bands, response linearity and noise measurements were made using a commercially available (Electro-Optical Industries, Inc), 10-inch square aperture, precision laboratory blackbody and a liquid nitrogen target. The blackbody is operable between 15 and $50 \mathrm{C}$ with $0.001 \mathrm{C}$ temperature uniformity and stability. ISIR was placed so that the blackbody filled its field of view. It recorded image frames for approximately 5 minutes at each of six temperatures from 15 to $40 \mathrm{C}$. A cold $(77 \mathrm{~K})$ external calibration source was provided by a pan of liquid nitrogen placed directly on the floor beneath ISIR, and which ISIR viewed at nadir. (For all laboratory and flight measurements the 1-inch thick ZnSe window was in place.) Two-point calibrations, using the LN2 target and the ISIR onboard blackbody, were performed before and after recording frames at each of the six temperatures from 15 to $40 \mathrm{C}$. Each change in the external blackbody's temperature was preceded by a single point calibration using the ISIR onboard blackbody. Using the average of three frames at each temperature, the noise, gain and linearity of response of each pixel was determined.

The LIRIS MBA camera came with a table of pixel gains stored in memory. In analysis of all image data, however, pixel gains were recalculated from the most recent calibration cycle rather than using those stored in memory.

During the Shuttle flight, ISIR was mounted in the Hitchhiker canister on a shuttle bridge structure straddling the payload bay (Figure 3). ISIR was mounted to the bridge with its optical axis at right angles to the shuttle longitudinal and lateral axes, so that with the payload bay doors open and the shuttle in an inverted attitude, it was nadir viewing. The shuttle flew a high-inclination orbit (57 
degrees) during the STS-85 mission. The orthogonal axes of the focal plane were aligned to within 0.1 degrees of a predetermined angle with the shuttle longitudinal and lateral axes. This angle helped to compensate in part for the lateral image smearing caused by the earth's rotational motion, a significant source of potential image smearing at high latitudes.

In TDI each image was built up in band-sequential manner with the filter wheel rotating. The success of TDI depends in part on the accuracy with which the same ground area can be sampled by the pixels whose signals are co-added. The requirement was to obtain less than $1 / 8$ of a pixel smear in our highest resolution mode. The stability of the shuttle platform is very important in this respect; shuttle roll, pitch and yaw cause misregistration of pixels in TDI. The velocity/height ratio $(\mathrm{V} / \mathrm{H})$ of the platform must also be related to the frame rate at which successive images are acquired. The camera provided by LIRIS operates at a fixed $60 \mathrm{~Hz}$ frame rate. In order to match the frame rate to the Shuttle $\mathrm{V} / \mathrm{H}$, the approach was to discard every other frame to achieve an effective $30 \mathrm{~Hz}$ frame rate. Hence although 33.34 milliseconds were required to acquire each image frame, the actual integration time was only 16.67 milliseconds. In order to minimize image smearing during the TDI-40 imaging experiments, the shuttle had to be at $141+/$ - 3 nautical miles altitude in order to match the $\mathrm{V} / \mathrm{H}$ to the frame rate. Second, the shuttle had to execute yaw correction maneuvers to compensate for the earth's rotational motion, because the tangential velocity of the IFOV resulting from the earth's rotation varies with latitude, hence it could not be completely eliminated by a single angular correction to the orientation of the focal plane. These measures resulted in image smear in TDI-40 of less than 0.125 IFOV.

During the STS-85 mission, ISIR was afforded the opportunity, via many shuttle attitude adjustment required to support the other 14 experiments on STS-85, to view deep space just prior to and just after earth-viewing datatakes. These views of space provided a uniform low temperature target for a two-point calibration. The calibration method adopted was to use the deep space view and the onboard black body at ambient temperature to perform two-point calibrations every 30 to 60 minutes, bracketing each data-take. Then during the data-take, onepoint calibration scenes were performed every five minutes using only the onboard blackbody.

By means of the two-point space calibrations the pixel gains and offsets were updated before each data run. The one-point calibrations that bracketed the data takes at fiveminute intervals were used to do a linear interpolation of the pixel offsets for images acquired during each data take. The brightness temperatures were obtained from these offsets and gains.

The design and execution of the imaging experiments, carried out with ISIR on the shuttle STS-85 mission, has been previously described by Spinhirne [4]. During the flight mission a total of 58 hours of earth viewing imagery were obtained. Typically the data were acquired in blocks of 2 to 4 hours of continuous operation. Of the data over ten hours were with shuttle attitude control to allow test of the high spatial resolution imaging. The other along track imaging were with flight parameters sufficient for $1 / 2$ pixel control on image smear. All of the along track imagery was in conjunction with SLA to provide direct laser cloud height measurements. Four hours of operation were acquired with the instrument operating in direct frame mode rather than TDI to test stereo imaging.

\section{ANAL ysis of PERFormance}

The microbolometer detector used in the first hitchhiker experiment was an early test unit. The detector had a significant numbers of defective pixels, approaching $30 \%$, and had less uniformity and greater noise than later and current models of the detector. In spite of this adequate results were obtained from laboratory and flight testing. The microbolometer array used in ISIR contained two bad rows near the center of the field of view, and two bad rows at the very top of the frame. There were no "out" columns. There were defective pixels: 6018 (or 8.3\% of 78480 pixels) are "dead" (permanently pegged at the middle of the range), and another 480 are "hot", or pegged at the top of the range. A map of the bad pixels was created for use in image smoothing by nearest-neighbor averaging. It was also used to remove the bad pixels when array mean properties such as response, NEDT, gain and offset were calculated. Finally, a small number of pixels were neither dead nor hot but had pathologically low gain and these were also excluded from computation of array mean NEDT, gain and offset. In computing radiometrically calibrated brightness temperatures, no smoothing was done until after the brightness temperature at each pixel was calculated.

In TDI, some of the sub-pixels whose signals are coadded are dead. Because the location of these bad subpixels is fixed and known, their effect on the resulting TDI pixel is taken account of in analysis by multiplying each TDI pixel by an effective gain and offset. These gain and offset values are determined from the number of contributing bad sub-pixels. All noise analysis and calibration in TDI is done on images corrected this way.

During STS-85, for calibration runs it was possible to calculate the gains and offsets. After calibrations, earth-viewing data was acquired and calibrated with the gains and interpolated offsets.

The NEDT was found to be a function of scene temperature and spectral bandwidth, as expected. NEDT was also a function of the number of integrated frames and the number of pixels in TDI, up to 10 frames and 10 pixels. An unexpected result was that integrating larger numbers of frames and pixels did not result in improved NEDT: the 
SNR in TDI-40 was no lower than in TDI-10. This is thought to be due to correlated row and column noise, which does not improve with signal integration and averaging.

The response linearity of ISIR was evaluated in the single frame and TDI-40 modes. In TDI and integrated frame modes, the total integrated signal is divided by the number of pixels in TDI or the number of integrated frames, in order to keep the output signal near the middle of the range of data. For this reason, the response in singleframe and integrated modes is expected to be the same. In single frame mode, the response of ISIR to flux is found to be almost perfectly linear over the range of temperatures from 15 to $40 \mathrm{C}$. It is possible that radiation loading from the blackbody at the highest three temperatures caused an apparent very slight deviation from linearity at a $25 \mathrm{C}$ scene temperature. This could be a consequence of two things. First, the fast optics, which are needed to boost the total integrated signal flux to achieve the desired SNR, make the instrument susceptible to radiation loading from and to the scene. Second, for preflight data, no housekeeping temperatures were available to compute the instrument background flux. Only the scene flux was known, whereas response versus total flux would be expected to be linear.

The results of the test of linearity in the TDI-40 mode, was examined from post-flight laboratory measurements. The response at a target temperature of $77 \mathrm{~K}$ is included at six temperatures from 15 to $40 \mathrm{C}$. The response of ISIR over the range from $77 \mathrm{~K}$ to $313 \mathrm{~K}$ is very linear. The slope of the response line gives the gain, which in Band 4 is $1.5 \times 1010$ counts/Watt, and this is in agreement with values reported by the manufacturer of the array. The linearity of response over the range from 77 to $313 \mathrm{~K}$ justifies the use of a two-point calibration to find the gain, and the use of a one-point calibration to update the offset.

The overall performance analysis of the radiometer can be summarized as follows. The observed NEDT from pre and post flight testing were approximately two to four times the limit specified for the imaging requirement of 0.1 k. An NEP of approximately $40 \mathrm{pW}$ can be inferred and a $\mathrm{D}^{*}$ of approximately $10^{9} \mathrm{~cm}(\mathrm{~Hz})^{1 / 2} / \mathrm{W}$. The results are consistent with the quality limitation of the early preproduction detector used for the first ISIR flight mission. When translated to the much better MBA detectors as now available, the imaging radiometric accuracy requirement would be met. Calibration accuracy appear to be very good, to within $0.1 \mathrm{k}$, based on analysis to date. As stated above the largest contribution to the NEDT in the integration modes was from correlated row and column noise.

Band to band pixel registration for the TDI along track imaging is obtain in a much different manner than for older imagers where all band instantaneously view the same IFOV. To achieve the science objectives of the ISIR experiment, imagery is to be analyzed in four wavelength bands covering the spectral region of 8 to $13 \mathrm{um}$. Measurements in each of these bands are made sequentially with the aid of the filter wheel that rotates the desired filter into the optical path quickly enough so that contiguous imaging can be achieved. Since the different wavelengths are acquired sequentially, however, the registration of the data must be done in post-processing. From one sequence of TDI at one wavelength, a frame of data consisting of multiple lines of cross track pixels is acquired. Successive wavelengths form overlapping images. Each surface pixel will be observed at each wavelength but at slightly different times. The registration is accomplished using ground-track information provided for STS-85.

As stated before, orbit and attitude requirements must be met in order to avoid pixel smearing in TDI. Similar the multiple wavelength registration will be effected by less than ideal parameters. A possible drift of the image scene is most effected by a combination of the shuttle's motion and the Earth's rotation. To avoid cross track image drift due to the change of the yaw relative to the ground track motion vector as a function of latitude, he shuttle was operated in special steering-mode. In this mode, the shuttle yaw is continually adjusted to counter the changing contribution of the Earth's rotation with latitude to the apparent ground velocity, thereby maintaining the desired constant alignment with the ground velocity vector. The shuttle pointing was maintained to within $1 / 10$ degree. Hence, all motion of the cloud scene across the detector is in one direction and the shuttle ground-track data can be used to register the ISIR images. Using the mission elapsed time (MET), latitude, and the average ground speed between two images acquired with ISIR is determined and the amount by which the image has moved along track direction between wavelengths is calculated.

Another aspect important to the registration of the ISIR images is brought about by the oblateness of the Earth. Although the shuttle is in a circular orbit, the altitude of the shuttle above the surface changes by approximately $15 \mathrm{~km}$ twice per orbit. This change in altitude results in a change in the footprint of the detector and subsequently the speed at which a cloud scene drifts across the detector. The frame readout rate for the ISIR detector was fixed at $30 \mathrm{hz}$. However for exact surface registration the required frame rate ranged between 29 and $31 \mathrm{hz}$, depending upon where the shuttle was in its orbit. In the initial design requirement for the ISIR instrument a variable frame rate was planned, but could not be implemented in the first flight version. The effect of the altitude change an a fixed frame rate is a variable overlap between successive multiwavelength scenes. It affects the amount of overlap in subsequent images of a common wavelength greatly enough that it becomes an important consideration for contiguous imaging. The overlap varied by as much as 14 pixel rows twice per orbit. The fact that this varying overlap changes continually rather than discretely also has important consequences for the image 
registration. Interpolation of the image is necessary to avoid a systematic uncertainty. For the ISIR measurements this systematic uncertainty in the image registration dominates the uncertainty in the registration caused by shuttle instability. Overall for the initial ISIR test flight the pixel registration error will be as large as $1 / 2$ pixel which is larger than the typical $1 / 4$ requirement stated for operational imagers. A variable image frame rate would mitigate the effect.

\section{IMAGING RESULTS}

In spite of the limitations described above for the first flight of the MBA based ISIR instrument, data results appear very good and sufficient for science analysis. In Fig. 4 is shown

6. SUMMARY

7. REFERENCES

1. "Observation of the Earth and lts environment," $H$. J. Kramer, Springer-Verlag, Berlin, 1994.

2. "Remote Sensing of the Lower Atmosphere an Introduction," G. L. Stephens, Oxford University Press, New York, 1994.

3. Modis ref

4. "Micromachined Bolometer Arrays" R. A. Wood and N. A. Foss, Laser Focus World, 101-106, June 1993.

5. "The 27-28 October FIRE IFO Cirus Case Study: Spectral Properties of Cirrus Clouds in the 8-12 um Window," S. A. Ackerman, W. L. Smith, J. D. Spinhirne, and H. E. Revercomb, Mon. Wea. Rev., 118, 2377-2388, 1990. 